\title{
Toll-Like Receptors Mediated Signalings: Friends or Enemies?
}

\author{
Yan Zhang
}

Published online: 25 August 2013

(c) Springer Science+Business Media New York 2013

Toll-like receptors (TLRs) were first identified in the fly Drosophila melanogaster as genes important for the establishment of dorsal-ventral orientation during embryonic development [1]. At present, at least 11 types of TLRs in humans and 13 in mice have been reported [2]. TLRs, widely distributed in humans (TLR4 and MD-2 are expressed in the normal colonic epithelium; TLR2, 4, and 9 are expressed in normal gastric epithelium), are characterized by an extracellular leucine-rich repeat and a cytoplasmic domain similar to the structure of the interleukin 1 (IL-1) receptor family.

Besides pathogen-associated molecular patterns (PAMPs), TLRs also recognize some damage-associated molecular pattern (DAMP) proteins, including hyaluronic acid, high-mobility group box 1 (HMGB1), and heat-shock proteins. These molecules signal the presence of impending or actual danger passively released from stressed cells or degraded extracellular matrix to surrounding cells and tissues.

TLR activation triggers intracellular signaling cascades, inducing expression of numerous immune response genes. Although all TLRs activate similar signaling pathways, some TLRs trigger specific pathways dependent on identity of the associated cytoplasmic adapter molecule, such as MyD88. TLR signaling is primarily divided into MyD88dependent and MyD88-independent pathways. Nearly all TLRs, with the exception of TLR3, can modulate MyD88

Y. Zhang $(\square)$

Department of Gastroenterology, West China Hospital of

Sichuan University, Chengdu, Sichuan, China

e-mail: fengyixx@sohu.com signaling. TLR4 is the only receptor reported to activate MyD88-dependent and MyD88-independent signaling pathways [3]. The MyD88-dependent pathway leads to the activation of nuclear factor- $\kappa \mathrm{B}(\mathrm{NF}-\kappa \mathrm{B})$ transcription factors and downstream mitogen-activated protein kinases (MAPKs), such as extracellular signal-regulated kinase (ERK), p38, and c-Jun N-terminal kinase (JNK). The MyD88-independent pathway activates interferon (IFN)regulatory factor (IRF3), a component of the late phase of $\mathrm{NF}-\kappa \mathrm{B}$ activation.

TLRs signaling pathways are important in the development of inflammation, although their contributions appear discrepant. For example, in murine mucosal injury models such as ethanol-induced gastric injury, and in colitis experimental models, TLR4 deficiency augmented damage and worsened overall health. Yet, TLR4-deficient mice exhibited less hepatic damage in ischemia-reperfusion, nonalcoholic steatohepatitis, and fibrosis models [4, 5]. Although TLR9 deficiency may protect mice from chronic DSS colitis, TLR9 ${ }^{-1-}$ mice are also more susceptible to T cell mediated colitis [6]. Although TLR4, TLR9, and TLR3 agonists can prevent mice from developing colitis, enhancement of TLR4 reactivity and TLR9 agonists worsen outcomes in experimentally-induced colitis and in liver ischemia/reperfusion injury [4, 7-9].

At present, the discordant effects of TLR activation on inflammation and injury are unexplained. In this issue, Ye et al. [10] reported that gastric mucosal injury (ethanolinduced gastric mucosa injury) was more severe in $\mathrm{TLR}^{-I-}$ mice and in mice overexpressing TLR4, suggesting that TLR4 expression must be maintained in a narrow range in order to maintain gastric mucosal homeostasis. In health, TLR4 signaling may induce the production of protective factors Cox-2 and $\mathrm{PGE}_{2}$ necessary for the preservation of gastric mucosal barrier integrity 
[11]. Nevertheless, systemic administration of exogenous TLR agonists may increase apoptosis while decreasing the proliferation and migration of epithelial cells, impairing restitution of the epithelium, while also altering immune cell recruitment and cytokine production, exaggerating the immune responses that contribute to severe tissue damage.

Although Ye et al. provided strong evidence that TLR4 is important for gastric mucosal homeostasis, the involvement of TLRs in the induction and progression of inflammation-related disease is still perplexing. Before TLRactive molecules can be used clinically, their contributions to immune activation, mucosal injury, and epithelial proliferation and apoptosis need to be carefully evaluated.

In conclusion, Ye's study showed an interesting phenomena that both deficient and excessive expression of TLR4 promotes ethanol-induced gastric mucosal injury. The underlying mechanism involves the MyD88/NF- $\mathrm{KB}$ signaling pathway and the HMGB1. Ye's paper may also provide an answer to the different results in some papers. According to this paper, TLR exhibits protective role in a very narrow range. Once the expression of TLR fits in this range, no matter what was used as an antagonist or an agonist, the inflammation can be alleviated. Otherwise, the injury will be aggravated. However, whether this phenomenon can be found in other organs like the colon, what is the exact bad effect of excessive expression of TLR4 on the stomach epithelium, and what is the exact safe range of TLRs all need to be clarified.

\section{Conflict of interest None}

\section{References}

1. Hashimoto C, Hudson KL, Anderson KV. The toll gene of Drosophila, required for dorsal-ventral embryonic polarity, appears to encode a transmembrane protein. Cell. 1988;52:269-279.

2. Sheedy FJ, O'Neill LAJ. The troll in toll: mal and tram as bridges for TLR2 and TLR4 signaling. J Leukoc Biol. 2007;82:196-203.

3. Akira S. Toll-like receptor signaling. J Biol Chem. 2003;278: 38105-38108.

4. Kang JW, Koh EJ, Lee SM. Melatonin protects liver against ischemia and reperfusion injury through inhibition of toll-like receptor signaling pathway. J Pineal Res. 2011;50:403-411.

5. Csak T, Velayudham A, Hritz I, et al. Deficiency in myeloid differentiation factor- 2 and toll-like receptor 4 expression attenuates nonalcoholic steatohepatitis and fibrosis in mice. Am J Physiol-Gastrointest Liver Physiol. 2011;300:G433-G441.

6. Lee J, Mo JH, Katakura $\mathrm{K}$, et al. Maintenance of colonic homeostasis by distinctive apical TLR9 signalling in intestinal epithelial cells. Nat Cell Biol. 2006;8:1327-1336.

7. Dong L, Li J, Liu Y, et al. TLR2mAb or/and TLR4mAb increase counts of Lactobacilli and Bifidobacteria in dextran sulfate sodium-induced colitis in mice. J Gastroenterol Hepatol. 2011;27:110-119.

8. Obermeier F, Dunger N, Deml L, et al. CpG motifs of bacterial DNA exacerbate colitis of dextran sulfate sodium-treated mice. Eur J Immunol. 2002;32:2084-2092.

9. Obermeier F, Dunger N, Strauch UG, et al. Contrasting activity of cytosin-guanosin dinucleotide oligonucleotides in mice with experimental colitis. Clin Exp Immunol. 2003;134:217-224.

10. Ye H, Hua R, Yu L, et al. Abnormal expression of toll-like receptor 4 is associated with susceptibility to ethanol-induced gastric mucosal injury in mice. Dig Dis Sci. (Epub ahead of print). doi:10.1007/s10620-013-2727-5.

11. Zhang Y, Chen H, Yang L. Toll-like receptor 4 participates in gastric mucosal protection through Cox-2 and PGE2. Dig Liver Dis. 2010;42:472-476. 